\title{
Questes
}

\section{La règle du jeu-parti : une autre thématique que l'amour courtois, la théorie des cinq sens}

\section{Midoriko Kageyama}

\section{(2) OpenEdition}

Édition électronique

URL : http://journals.openedition.org/questes/667

DOI : 10.4000 /questes. 667

ISSN : 2109-9472

Éditeur

Les Amis de Questes

\section{Édition imprimée}

Date de publication : 15 février 2010

Pagination : 65-77

ISSN : 2102-7188

\section{Référence électronique}

Midoriko Kageyama, «La règle du jeu-parti : une autre thématique que l'amour courtois, la théorie des cinq sens », Questes [En ligne], 18 | 2010, mis en ligne le 01 janvier 2014, consulté le 20 avril 2019. URL : http://journals.openedition.org/questes/667 ; DOI : 10.4000/questes.667 


\title{
La règle du jeu-parti : une autre thématique que l'amour courtois, la théorie des cinq sens
}

\author{
Midoriko KAGEYAMA
}

Héritier du partimen des troubadours ${ }^{1}$, le jeu-parti se développe durant la seconde moitié du XIII ${ }^{\mathrm{e}}$ siècle dans le Nord de la France. Comme Arthur Långfors le constate, le sujet généralement abordé est celui des « demandes d'amour $»^{2}$. Michèle Gally discute des topoi principaux fondés sur l'amour courtois descendant lui-même de la fin'amors, tels que le motif printanier et la description de la dame ${ }^{3}$. Le sujet du jeu est souvent qualifié $\mathrm{d}^{\prime}$ « homogène $)^{4}$.

Ce jeu, consacré à l'origine au divertissement de la cour en Provence, fleurit dans la société bourgeoise d'Arras. Autrement dit, il devient un jeu « pour le peuple $»^{5}$, si l'on se réfère à l'expression d'Alfred Jeanroy sur la poésie lyrique des trouvères. Même les marchands et les banquiers y participent en tant que poètes amateurs ${ }^{6}$. Le jeu-parti du Nord de la France,

\footnotetext{
1 Alfred JEANROY, Les Origines de la poésie lyrique en France au Moyen Âge. Études de littérature française et comparée, suivies de textes inédits, Paris, Librairie Hachette, 1889, p. 46-47. Jeanroy suppose que le partimen pènetre par le Limousin et le Poitou dans la France du Nord.

2 Arthur LÅngfors, Alfred JEAnRoy et Louis Brandin, Recueil Général des Jeux-Partis Français, t. 1, Paris, Librairie Ancienne Édouard Champion, 1926, p. IX.

3 Michèle GALLY, Rhétorique et histoire d'un genre: le jeu-parti à Arras, Lille-thèses, 1985, p. 49-50.

4 Arthur LÅngfors, Alfred JEAnRoy et Louis Brandin, Recueil Général des Jeux-Partis Français, op. cit., p. IX.

5 Alfred JEANROY, Les Origines de la poésie lyrique en France au Moyen Âge, op. cit., p. XI.

6 Michèle Gally, Rhétorique et histoire d'un genre: le jeu-parti à Arras, op. cit., p. 106-109. Nous nous référons également aux études historiques, comme celle de Marie UnguREANU, La Bourgeoisie naissante: société et littérature bourgeoises d'Arras aux XII et XIII siècles, Arras, Commission des monuments historiques du Pas-de-Calais, 1955, p. 97.
} 
étant influencé par l'enseignement des exercices de la dialectique scolastique, renforce le raisonnement par la controverse ${ }^{7}$.

Les règles formelles principales du jeu-parti ${ }^{8}$ sont les suivantes. Une pièce ordinaire est composée de six strophes suivies de deux envois (parfois manquants). Les partenaires sont en général deux. Dans le premier couplet, l'un des deux joueurs pose une question dilemmatique à l'autre. Le deuxième choisit l'une des deux propositions dans la deuxième strophe. Le premier prend la proposition restante. Dès lors, ces deux joueurs défendent chaque proposition alternativement jusqu'à la sixième strophe. Chaque strophe commence par le nom et le statut de chaque joueur. Dans les deux envois, chacun choisit un juge, mais il n'apparaît pas et ne parle pas ${ }^{9}$. Ainsi, la discussion dans la pièce, en terminant sans conclusion ni sentence absolue, laisse le jeu ouvert.

Nous essayerons ici de proposer une relecture de deux pièces qui associent la sagesse et la grossièreté, dans et hors de la cour, afin de découvrir les nouveaux codes du jeu-parti. Ces deux pièces traitent tout particulièrement de la question des cinq sens, qui témoigne d'une ouverture du jeu-parti aux problèmes philosophiques. La vue, l'ouïe, l'odorat, le goût et le toucher, dont la description repose sur les définitions antiques, notamment aristotéliciennes ${ }^{10}$, font l'objet d'une classification d'ordre

\footnotetext{
7 Alfred Jeanroy, Les Origines de la poésie lyrique en France au Moyen Âge, op. cit., p. 46.

8 Arthur LÅngfors, Alfred Jeanroy et Louis Brandin, Recueil Général des Jeux-Partis Français, op. cit., p. V-VI. et Michèle GALLY, Rhétorique et histoire d'un genre: le jeu-parti à Arras, op. cit., p. 34-39.

${ }^{9}$ Le texte est généralement accompagné de musique.

${ }^{10}$ Voir ARISTOTE, De sensu et sensato, 2 (436a-438b), trad. anonyme (ou de NiCOLAS DE RegGIO), textus editionis quae paratur a L. Peeters (Aristoteles Latinus, XIII,1), De historia animalium, 4 (532b-537b), trad. GUILLAUME DE MOERBEKE, Pieter BEULLENS et Fernand Bossier (éd.), 2000 (Aristoteles Latinus, XVII, 2-I-1), De partibus animalium, 2
} 
physiologique, philosophique et théologique au Moyen Âge.

\section{La hiérarchie des cinq sens au XIII ${ }^{\mathrm{e}}$ siècle}

La hiérarchie de cinq sens est instable à cette époque. ${ }^{11}$ Tantôt la vue est valorisée, ou bien l'ouie ; tantôt le goût est privilégié, ou le toucher. Selon les critères adoptés, tels que la morale et le plaisir de l'amour, la hiérarchie varie. C'est un système qui compose «un dispositif complexe, matrice de multiples problématiques qui intéressent aussi bien le corps que l'âme, la philosophie que la littérature, la médecine que la théologie $»^{12}$, comme en témoignent de multiples traductions et commentaires consacrés spécifiquement à la théorie d'Aristote concernant les cinq sens.

Dans Li Livres dou Tresor de Brunet Latin, chaque sens est classé en fonction de sa position sur le visage. En général, la vue, l'ouïe, l'odorat, le goût et le toucher correspondent respectivement aux yeux, aux oreilles, au nez, à la bouche ${ }^{13}$ et aux mains. La vue se situe au sommet de cette hiérarchie, suivie par l'ouïe, l'odorat et le goût :

«Mais li cors a .V. autres sens, veoir, oïr, odorer, gouster, touchier. Et si come li uns devancist l'autre par honorableté de son estage, tout autresi devancist il l'un de l'autre de vertu. Car flerier sormonte au goster et de leu et de viertu, por ce k'il est plus en haut et oevre sa vertu plus de long. Autresi l'oreille sormonte au flerier, car nous oons plus de long ke nous ne flerons. Et le veoir les sormonte

(655b-662b), trad. GUILlAUME DE MOERBEKE, textus editionis quae paratur a Pietro RosSI (Aristoteles Latinus, XVII, 2-4), De anima, 2 (416a-424b), trad. JACQUES DE VENISE, textus editionis quae paratur a Jos DeCORTE et Josef BRAMS (Aristoteles Latinus, XII, 1) et Metaphysica, 1 (980a-980b), trad. anonyme (zu 'media'), Gudrun VuILLEMIN-DiEM (éd.), 1976 (Aristoteles Latinus, XXV, 2).

11 Jacqueline Cerquiglini-Toulet, «Le schéma des cinq sens d'une théorie de la connaissance à la création de formes littéraires », Micrologus, t. 10, Florence, Sismel et Editioni del Galluzo, 2002, p. 55-69.

12 Jean-Marie Fritz, Paysages sonores du Moyen Âge. Le versant épistémologique, Paris, Honoré Champion, 2000, p. 15.

${ }^{13}$ La bouche peut signifier également la parole. Nous aborderons cet aspect plus loin. 
trestous, et de leu et de dignité. $)^{14}$

On valorise davantage les deux sensations visuelle et auditive en contexte philosophique, scientifique ou théologique ${ }^{15}$. Nous nous contentons ici de citer un passage du Bestiaire d'Amor :

«Ensi fui je pris l'oïr et au veoir : dont ne fu che mie merveille se je perdi mon sens et memoire, car oïrs et veoirs sont les deus portes de memoire, si comme il a esté devant dit, et si sont ore doi des plus nobles sens de l'home. " ${ }^{16}$

Par ailleurs, de même que la vue et l'ouïe forment une paire, le goût et le toucher en forment une autre. L'antagonisme entre les deux paires est discuté depuis l'Antiquité. S'agissant des délices et du désir, le goût et le toucher sont supérieurs aux trois autres sens et plus puissants que les autres :

« Delis et desiriers sont acomplis et mis en oevre par les .V. sens dou cors, dont gouster et touchier sont principaus. Mais li autre troi sont establi pour ces .ii. ; car nos cognoissons la chose de long par veoir et par oïr et par flerier, ce ke le gouster ne touchier ne puet connoistre se de prés non [...] Nous lisons el livre de la Nature as Animaus que touchier et goster sont plus poissans en l'ome que en nule autre beste ; mais le veoir et l'oïr et le flairier sont de menour pooir en l'ome que es autres animaus. Et por çou ki jou ke li delit ki sont pour touchier et pour gouster sont plus perilleus des autres, et les vertus ki sont lor contraires sont de grignor vaillance. ${ }^{17}$

C'est ainsi que cette opposition donne matière à une discussion.

14 BrunetTo Latini, Li Livres dou Tresor, Francis James CARMODY (éd.), Los Angeles, Barkeley, 1948 (réédition, Genève, Slatkine Reprints, 1998), Livre I, ch. XV, p. 29. Ce passage est cité dans l'article de Jacqueline Cerquiglini-Toulet, « Le schéma des cinq sens d'une théorie de la connaissance à la création de formes littéraires », art. cit., p. 64.

15 Jean-Marie Fritz analyse cet aspect en se référant aux œuvres de Richard de Fournival, Alberd le Grand et Thomas d'Aquin. Cf. Paysages sonores du Moyen Âge, op. cit., p. 19. Par ailleurs Jean-Louis Chrétien traite de l'unité de la vision et de l'audition, en la rapportant au cœur. Cf. Jean-Louis ChretIEN, Symbolique du corps. La tradition chrétienne du Cantique des Cantiques, Paris, P.U.F., 2005, p. 21-22.

16 Richard De Fournival, Le Bestiaire d'Amour et la Response du Bestiaire, Gabriel BiAnCiOTto (éd.), Paris, Honoré Champion, 2009, p. 200.

17 BRUnetTo LATINI, Li Livres dou Tresor, op, cit., Livre II, ch. LXXIII, p. 249. 


\section{L'antagonisme courtois entre les sensations nobles et la sensation tactile}

Une pièce de Thibaut de Champagne, roi de Navarre, discute de l'antagonisme entre ces deux paires (RS 1393, linker 215-7) ${ }^{18}$. Wallensköld suppose, en tenant compte du partenaire de Thibaut qui marche avec une béquille, que cette pièce a été composée après 1240 ou $1243^{19}$. Tout d'abord, son partenaire Raoul propose un débat à Thibaut de Champagne :

« Sire, loez moi a choisir

D'un jeu, li quiex doit mielz valoir

Ou souvent s'amie sentir,

Baisier, acoler, sanz veoir, Sanz parler et sanz plus avoir

A toz jors mès de ses amors,

Ou veoir et parler toz jors,

Sanz sentir et sanz atouchier.

Se l'un en covient a laissier,

Ditez li quiex est mains joians

Et dou quel la joie est plus granz. $»^{20}$

«Veoir» et « parler » sont comparés aux verbes « sentir » et « atouchier ».

Thibaut justifie l'importance de « veoir » et « parler» dans le couplet suivant

18 Cette pièce a vu le jour en 1742 (éd. LeVESQUe DE LA RAVALliERE). Nous nous référons ici aux deux éditions d'Arthur Långfors et d'Axel Wallensköld. Cf. Arthur LÅngFors, Alfred JeANROY et Louis Brandin, Recueil Général des Jeux-Partis Français, op. cit., p. 29-33. et Les chansons de Thibaut de Champagne, roi de Navarre, Axel WALlENSKÖLD (éd.), Paris, Librairie Ancienne Édouard Champion, 1925, p. 147-152.

${ }^{19}$ Le titre de roi adressé à Thibaut conduit Wallensköld à penser que cette pièce a été composée après son avènement en 1234. De plus, il suppose que Raoul marche avec une béquille en raison des trois mots : "potence» (v. 42), «baston» (v. 47) et «bordon» (v. 55). Cela le conduit à dater ce jeu-parti d'après la croisade du roi Thibaut (1239-40) et même après le retour de Raoul en France (1243). Il mentionne aussi l'opinion de Winkler qui place la rédaction du poème entre 1252 et 1253. Les chansons de Thibaut de Champagne, roi de Navarre, Axel WALLENSKÖLD (éd.), op. cit., p. 150-151.

20 Arthur LÅngFORS, Alfred JEANROY et Louis BRANDIN, Recueil Général des Jeux-Partis Français, op. cit., p. 29, v. 1-11. Nous consultons également la traduction en français moderne, THIBAUT DE CHAMPAGNE, Recueil de Chansons, traduit par Alexandre MiCHA, Paris, Klincksieck, 1991, p. 105-106. 
en y substituant « bel ris » et « solacier $»^{21}$, lesquels allègent la souffrance. Raoul réplique en faisant référence à une caractéristique corporelle de son adversaire :

« Sire, vos avés mout bien pris

De vostre amie resgarder, Que vos ventres gros et farsis

Ne porroit soffrir l'adeser;

Et por ce amés vos le parler

Que vos solas n'est preuz aillors. » ${ }^{22}$

Le vers «vos ventres gros et farsis» fait allusion à l'embonpoint que Thibaut de Champagne se reconnaît lui-même ${ }^{23}$. Il est obligé de s'abstenir de toucher sa dame parce que son ventre l'en empêche. Ce passage nous montre que « veoir » et « parler » constituent un acte distancié de l'objet. Dans le couplet suivant, « parler» est remplacé par « oïr ${ }^{24}$. Nous nous rappelons cette phrase dans Li Livres dou Tresor: « nos cognoissons la chose de long par veoir et par oïr et par flerier, ce ke le gouster ne touchier ne puet connoistre se de prés non. $\gg{ }^{25}$.

Le roi de Navarre insiste sur le fait que c'est la béquille de Raoul au lieu de son gros ventre qui l'empêche d'embrasser sa dame dans l'obscurité, puisqu'elle est privée de la vue ${ }^{26}$. C'est ainsi qu'ils se moquent de leurs

21 Arthur LÅngFORS, Alfred JEANROY et Louis BRANDIN, Recueil Général des Jeux-Partis Français, op. cit., p. 30, v. 16-20. « Més quant il ne puet remenoir, / Ou veoir a plus de secors / Et ou parler qui est d'amors ; / Si bel ris et si solacier / Feront ma dolor alegier, $[\ldots] »$.

22 Ibid., p. 30. v. 23-28.

${ }^{23}$ Ibid., p. 31-32, v. 44 : « Que de mon gras ventre farsi ».

${ }^{24}$ Ibid., p. 31, v. 38 et v. 62.

25 BrunetTo Latini, Li Livres dou Tresor, op, cit., Livre II, ch. LXXIII, p. 249.

26 Arthur LÅngFoRs, Alfred JEANROY et Louis BRANDIN, Recueil Général des Jeux-Partis Français, op. cit., p. 30, v. 37 - p. 31, v. 44. «La ou l'en ne puet alumer, / Veoir, oïr, joie mener, / L'en n'i doit avoir fors que plors ; Et s'ele met sa main aillors / Quant vos cuidera embracier, / Se la potence puet baillier, / Plus avra duel, je vous afi, / Que de mon gros ventre farsi ». 
défauts physiques. Un savoir sur les cinq sens coexiste avec une plaisanterie.

Outre le mot «atouchier», Raoul emploie plusieurs termes tels que «adeser ${ }^{27}$, « baisier $», ~ «$ acoler $»$, et $«$ embracier ${ }^{28}$. Parmi eux, on pourrait considérer «baisier» comme un mot renvoyant au goût ou à la bouche. Nous remarquons ici que figurent dans cette pièce deux verbes, «parler ${ }^{29}$ dans les propos de Thibaut de Champagne et «baisier» dans ceux de Raoul, se rapportant à la bouche. En fait, les deux mots s'opposent. Tibaut souligne le grand secours et la consolation de la douleur par le fait de «parler ${ }^{30}$. En le contredisant, Raoul critique les belles paroles par les deux expressions : « faus plaideors » et « mençongier» :

«Einsi va des faus plaideors,

Dont li semblant sont mençongier.

Mez d'acoler et de baisier

Fet bone dame a son ami

Cuer large et loial et hardi. $»^{31}$

Le « baisier » est rapporté aux qualités de chevalier. «Cuer » signifierait ici une disposition de l'esprit proche du courage. Toutefois, dans le dernier couplet, Thibaut associe ce mot «cuer» à un verbe «catoillier» (« chatouiller») lié à l'idée de «toucher». En bref, il s'introduit dans le langage de son adversaire et tente de rapprocher leurs points de vue. On peut considérer que « cuer» a alors plutôt le sens de « sentiment ».

« Mès mout vaudroit mielz en amors

Veoir et oïr qu'estre aillors,

Rire, parler et solacier

27 Ibid., p. 30, v. 26.

28 Ibid., p. 31, v. 52.

${ }^{29}$ Ibid., p. 30, v. 18 et p. 32, v. 63.

${ }^{30}$ Ibid., p. 30, v. 17-20. « Ou veoir a plus de secors / Et ou parler qui est d'amors ; / Si bel ris et si solacier , / Feront ma dolor alegier... ».

${ }^{31}$ Ibid., p. 30. v. 29-33. C'est nous qui soulignons. 
Douz moz, qui font cuer catoillier,

Et resjoïr et saouler

Que en tenebres tastoner. $)^{32}$

Ainsi se déroule le débat dans cette pièce contenant parfois des digressions, et qui évite l'antagonisme définitif. On pourrait y retrouver en un certain sens l'esprit courtois propre à la cour du roi. Mais ce n'est pas toujours le cas en dehors de la cour. Nous allons examiner une autre pièce où une argumentation plus rigoureuse porte également sur la théorie des cinq sens.

\section{Le débat sur cinq sens in absentia}

La pièce suivante, qui a été étudiée par Alfred Jeanroy en tant que jeu-parti en $1897^{33}$, figure dans le Recueil Genéral des Jeux-partis français (1926) (RS 25; Linker 247-1) . $^{34}$ Un des deux joueurs de cette pièce est Colard le Changeur qui traite du thème du moine et de la nonne dans d'autres pièces. Jeanroy suppose qu'en 1897, il est clerc. Cette pièce provient de l'école bourgeoise d'Arras, et date de la deuxième moitié du $\mathrm{XIII}^{\mathrm{e}}$ siècle $^{35}$. Dans le premier couplet, une question dilemmatique est posée par le premier interlocuteur, Sandrart :

« Doy home sont auques tout d'un eage

Qui par amours ainment bien loiaument

Une dame, qui est plaissans et sage,

Dont ainc nulz d'iaus ne gehi son talent.

Or leur avient, par fortune contraire,

Que li uns pert les ieus de son viaire,

${ }^{32}$ Ibid., p. 31, v. 61 - p. 32, v. 66.

33 Alfred JEANROY, « Jeux partis inédits du XIII" siècle », Revue des langues romanes, $4^{\mathrm{e}}$ série, t. 10 (1897), p. 350-367. Ce jeu-parti a été inédit avant 1897.

34 Arthur LÅngFors, Alfred JEANROY et Louis BRANDIN, Recueil Général des Jeux-Partis Français, op. cit., p. 58-61.

35 Alfred JEANROY, « Jeux partis inédits du XIII ${ }^{\mathrm{e}}$ siècle », art. cit., p. 350-351. 
Et li autres amuïst nüement ;

De leur desirs n'amenrissent noient,

Ainz weut chascuns son pourpos poursuivir.

Li quex en a le plus bel pour joïr? $»^{36}$

Deux vassaux de condition presque identiques au départ aiment la même dame. Montrer au début d'un jeu-parti la situation similaire de deux amants ou amantes, c'est la règle du jeu. Dans cette pièce, l'un devient aveugle par une mauvaise fortune, l'autre sourd et muet. À cette époque-là, les mots «aveugle $»^{37}$, «sourd $»$ et $«$ muet ${ }^{38}$ sont parfois connotés et revêtent un sens négatif, renvoyant à la perte de facultés. Mais ici, les joueurs défendent les autres sens et capacités s'exerçant à la place des facultés absentes. En témoigne la seconde strophe :

«Cilz doit avoir trop plus le cuer dolent

Qui riens ne voit ne ne sent, s'il ne flaire,

Que cilz qui voit : car tout par essemplaire

Moustre mout bien auques que ces cuers sent.

S'il ne parole, s'a il par vir talent

Par quoi il doit les biens d'amours sentir

Plus c'uns parlens qui onques ne peust vir. $\gg{ }^{39}$

Dans cette pièce, quatre des cinq sens sont convoqués : la vue (« vir ») naturellement, l'odorat (« s'il ne flaire »), le toucher (« touchier $»^{40}$ )

\footnotetext{
36 Arthur LÅngFors, Alfred JEANROY et Louis BRANDIN, Recueil Général des Jeux-Partis Français, op. cit., p.58, v. 1-10.

37 On dépeint souvent l'aveugle au XIII ${ }^{\mathrm{e}}$ siècle comme un mendiant vicieux. Dans la farce Le Garçon et l'Aveugle, un aveugle est trompé par un valet à la fin. Cf. Jean Dufournet (éd.), Le Garçon et l'Aveugle, Paris, Honoré Champion, 2005, p. 130-151.

38 Dans les œuvres de Chrétien de Troyes, «muet» et «sourd» ont parfois une connotation dépréciative: «Tant set et tant puet et tant vaut / Mes sire Keus an totes corz / Qu'il n'i ert ja müez ne sorz. / Bien set ancontre vilenie / Respondre san et corteisie [...]», Chretien de Troyes, Le Chevalier au lion (Yvain), Halle, 1887, v. 632-637.

39 Arthur LÅngFors, Alfred JEANROY et Louis BRANDIN, Recueil Général des Jeux-Partis Français, op. cit., p. 59, v. 16-22.

${ }^{40}$ Ibid., p. 59, v. 24.
} 
et l'ouïe (« ouïr $»^{41}$ ). Tout d'abord Colart insiste sur la supériorité de la vue dans une dimension philosophique et juste après dans une théorie sur l'amour. Dans la strophe suivante, Sandrart s'oppose à Colart à propos d'un argument grossier.

«Car, s’on vouloit, on li feroit bessier

Tel estrement ou ne devroit touchier.

Certes, Colart, pou quiert son aventaige

Cilz qui el bien veult metre abaissement $\gg{ }^{42}$

Il soutient la supériorité de la parole sur la vue dans la théorie sur l'amour et dévalorise le toucher. Le verbe « moustrer » correspond à celui qui est employé par Colart ${ }^{43}$ :

« Cilz par son vir puet avoir l'ueil au vent,

Et li parlers moustre le sentement

C'on a el cuer, dont naissent li souspir

Qui deus vouloirs fait tout un devenir. »> ${ }^{44}$

Nous remarquons ici que les deux joueurs associent souvent la parole à l'un des cinq sens, par exemple à la vue, comme le souligne le vers suivant : « S'il ne parole, s'a il par vir talent ${ }^{45} »$. Dans la cinquième strophe, le «parler » et l'« ouïr » sont directement juxtaposés ${ }^{46}$. Dès lors, on peut penser que les deux joueurs considèrent la parole comme le cinquième sens remplaçant celui du goût.

${ }^{41}$ Ibid., p. 60, v. 52.

${ }^{4}$ Ibid., p. 59, v. 23-26.

${ }^{43}$ Ibid., p. 59, v. 19.

${ }^{44}$ Ibid., p. 59, v. 31-34.

45 Ibid., p. 58, v. 20.

${ }^{46}$ Ibid., p. 60, v. 52. 


\section{L'enjeu de la relation entre les cinq sens et le cour}

Ces deux joueurs discutent également de la relation entre la vue et le cœur et entre la parole et le cœur. L'opinion de Colart ${ }^{47}$ est que celui qui possède la vue peut voir comme en image tout ce que le cœur ressent. Selon son partenaire ${ }^{48}$, seul celui qui parle montre le sentiment qu'il a dans le cœur d'où naît le soupir. De plus, Colart soutient que le muet voit là où son cœur demeure :

"Que li muiaus qui voit ce en presant

U ses cuers gist. ${ }^{49}$

D'autre part, son interlocuteur défend l'idée que, par rapport au muet qui ne peut ni parler ni entendre, l'aveugle n'éprouve qu'un seul malheur, à savoir ne pas « voir par dehors » ${ }^{50}$. Puis, il poursuit « Qui u cuer se repaire,/ Li vient du cuer, ou bonne amour se prent $»^{51}$. Cela veut dire que ce qui s'en retourne au cœur lui vient du cœur où l'amour véritable s'allume. Comment comprendre cet argument? En fait, il y a deux manières de voir : la vue de l'extérieur du cœur et la vue de l'intérieur du cœur.

Jacqueline Cerquiglini-Toulet, dans son $\operatorname{article}^{52}$, citant le Chastel Perilleux de Robert le Chartreux, fait mention du château du cœur qui a cinq portes correspondant aux cinq sens. La bouche y est la porte principale avec la langue. Suivant cette «spatialisation », on pourrait dire que la vue et la

${ }^{47}$ Ibid., p. 59, v. 18-19. « [...] tout par essemplaire / Moustre mout bien auques que ces cuers sent. $»$.

48 Ibid., p. 59, v. 32-33.

49 Ibid., p. 60, v. 44-45.

50 Ibid., p. 60, v. 54.

51 Arthur LÅngfors, Alfred JEANROY et Louis Brandin, Recueil Général des Jeux-Partis Français, op. cit., p. 60, v. 54-55.

52 Jacqueline CerquiglinI-Toulet, «Le schéma des cinq sens d'une théorie de la connaissance à la création de formes littéraires », art. cit., p. 64-66. 
parole sont deux sortes de «portes» vers le cœur. En d'autres termes, la vue se situe au point de passage qui se dirige vers le lieu où le cœur demeure. D’ailleurs, la parole concrétise ce lieu afin de l'exprimer « avec la langue », soit en mots.

Sandrart fait allusion au regard qui porte sur l'intérieur du cœur ${ }^{53}$, alors que Colart donne davantage d'importance à la vue de l'extérieur jusqu'à la fin, comme en témoigne le dernier couplet :

«Chascun set bien que nus maus ne s'apere

Envers celui qui ne voit c'une aumaire

Qui est de bos, ne nes le sacrement

Ne puet il vir: dont je di que li cent

$\mathrm{Ne}$ valent pas le veüt, sans mantir. » ${ }^{54}$

L’occurrence du terme religieux «sacrement» dans ce contexte paraît incohérent. Mais, on pourrait comprendre que l'absence de la vue fait obstacle à l'aveugle dans la foi, tant la vue de l'extérieur est importante. Dans tous les cas, les deux joueurs placent le cœur au dessus des cinq sens.

Ainsi, le tournoi en vers de ces deux pièces ne franchit pas les frontières de l'amour courtois conformément à la règle du jeu. L'une en évitant la discorde, avance une discussion légère et globale sur les cinq sens,

53 Jean-Louis Chrétien, citant saint Augustin, parle d'une vision du cœur. Sa citation est traduite par Berrouard: "Reviens à ton coeur, et vois en lui ce que peut-être tu dois penser de Dieu, puisque c'est là que se trouve l'image de Dieu. » (Euvres de saint Augustin 72, Homélies sur l'Évangile de saint Jean, XVIII, 7, traduite par Marie-François Berrouard, Paris, Desclée de Brouwer, 1977, p. 137.). Si on substitue l'amour à Dieu, cela explique bien l'opinion de Sandrart, semble-t-il. Cf. Jean-Louis ChrEtiEN, Symbolique du corps. La tradition chrétienne du Cantique des Cantiques, op. cit., p. 21.

54 Arthur LÅngFORS, Alfred JEANROY et Louis BRANDIN, Recueil Général des Jeux-Partis Français, op. cit., p.60, v.65-69. Dans Somme Théologique de Thomas d'Aquin, il est question que l'objet de la foi soit une chose vue. Cf. THOMAS D'AQuIN, Somme Théologique, éd. traduite par Antonin-Marcel HENRY, Carlos-J. PINTO DE Oliveira, Albert Raulun, Aimon-Marie Roguet et Jean-Pierre Torrell, Tome 3, II-II, Paris, Cerf, 1985, Question 1, article 4, p. 21-22. 
l'autre approfondit la question des cinq sens jusqu'à la relation avec le cœur qui évoque une disputatio sur la pensée d'Aristote ou de saint Augustin. On peut alors se demander si le sujet du jeu-parti était limité. Les poètes semblent plutôt préférer argumenter autour d'une autre thématique, comme ce sujet philosophique, en le dissimulant derrière une théorie de l'amour répandue et accessible à tous les états sociaux. 